\title{
Increased extracellular volume in the liver of pediatric Fontan patients
}

\author{
Charlotte de Lange ${ }^{1,2}$, Marjolein J. E. Reichert ${ }^{1}$, Joseph J. Pagano ${ }^{3}$, Mike Seed ${ }^{1,4}$, Shi-Joon Yoo ${ }^{1,4}$, Craig S. Broberg ${ }^{5}$, \\ Christopher Z. Lam ${ }^{4}$ and Lars Grosse-Wortmann ${ }^{1,4^{*}}$ (D)
}

\begin{abstract}
Background: Patients with single ventricle physiology are at increased risk for developing liver fibrosis. Its extent and prevalence in children with bidirectional cavopulmonary connection (BCPC) and Fontan circulation are unclear. Extracellular volume fraction (ECV), derived from cardiovascular magnetic resonance (CMR) and T1 relaxometry, reflect fibrotic remodeling and/or congestion in the liver. The aim of this study was to investigate whether pediatric patients with single ventricle physiology experience increased native T1 and ECV as markers of liver fibrosis/congestion.
\end{abstract}

Methods: Hepatic native T1 times and ECV, using a cardiac short axis modified Look-Locker inversion recovery sequence displaying the liver, were measured retrospectively in children with BCPC- and Fontan circulations and compared to pediatric controls.

Results: Hepatic native T1 time were increased in Fontan patients ( $n=62,11.4 \pm 4.4$ years, $T 1762 \pm 64 \mathrm{~ms}$ ) versus BCPC patients $(n=20,2.8 \pm 0.9$ years, T1 $645 \pm 43 \mathrm{~ms}, p=0.04)$. Both cohorts had higher T1 than controls $(n=44,13.7 \pm 2.9$ years, T1 $604 \pm 54 \mathrm{~ms}, p<0.001$ for both). ECV was $41.4 \pm 4.8 \%$ in Fontan and $36.4 \pm 4.8 \%$ in BCPC patients, respectively $(p=0.02)$. In Fontan patients, $T 1$ values correlated with exposure to cardiopulmonary bypass time $(R=0.3, p=0.02)$, systolic and end diastolic volumes ( $R=0.3, p=0.04$ for both) and inversely with oxygen saturations and body surface area $(R=-0.3, p=0.04$ for both). There were no demonstrable associations of $\mathrm{T} 1$ or ECV with central venous pressure or age after Fontan.

Conclusion: Fontan and BCPC patients have elevated CMR markers suggestive of hepatic fibrosis and/or congestion, even at a young age. The tissue changes do not appear to be related to central venous pressures.

Trial registration: Retrospectively registered data.

Keywords: Cardiovascular magnetic resonance, Fontan circulation, Liver cirrhosis, T1 mapping, Single ventricle

\section{Introduction}

The Fontan operation, introduced over 40 years ago, and its permutations place the systemic and pulmonary circulations in series rather than in parallel in patients with functionally univentricular hearts $[1,2]$. Fontan and bidirectional cavopulmonary connection (BCPC) hemodynamics depend on a central venous pressure that is high enough to overcome the pulmonary vascular resistance. The ensuing systemic venous congestion and a limited ability to augment cardiac output result in end-organ dysfunction,

\footnotetext{
*Correspondence: lars.grosse-wortmann@sickkids.ca

'Department of Pediatrics, Division of Cardiology, The Hospital for Sick

Children, University of Toronto, Ontario, Canada

${ }^{4}$ Department of Diagnostic Imaging, The Hospital for Sick Children, University of Toronto, Toronto, Ontario, Canada

Full list of author information is available at the end of the article
}

affecting the liver, intestine, and other organ systems [3] . Fontan associated liver disease (FALD) with hepatic congestion and fibrotic remodeling are increasingly recognized [4]. However, the extent of hepatic fibrosis as well as its potential causes and clinical significance are incompletely understood, partially due to the current difficulties in monitoring tissue fibrosis non-invasively.

Native T1 times and extracellular volume fraction (ECV) derived from cardiovascular magnetic resonance (CMR) T1 relaxometry, often referred to as 'T1 mapping, are non-invasive markers of diffuse tissue fibrosis increasingly applied to the myocardium [5-9]. More recently, CMR T1 mapping of the liver has been employed to quantify the severity of chronic liver disease in adults without congenital heart disease, yielding a good

(c) The Author(s). 2019 Open Access This article is distributed under the terms of the Creative Commons Attribution 4.0 International License (http://creativecommons.org/licenses/by/4.0/), which permits unrestricted use, distribution, and 
correlation to the histological severity of fibrotic remodeling [10-12].

In the present study we aimed to investigate the feasibility of T1 mapping in the liver in pediatric Fon$\tan$ and $\mathrm{BCPC}$ patients on routinely performed CMR. We hypothesized that CMR markers of extracellular volume (ECV) expansion are increased in patients with single ventricle physiology and associated with their hemodynamics.

\section{Methods}

In this retrospective cross-sectional cohort study, consecutive pediatric patients with $\mathrm{BCPC}$ and Fontan-type palliations who underwent CMR between April 2014 and March 2018 were included. During that interval T1 relaxometry was routinely included in the CMR protocol. Patients with a non-diagnostic T1 relaxometry acquisition pertaining to the liver were excluded. Only the latest exam was included when more than one CMR scan were eligible.

Demographic, clinical, and surgical Information were retrieved from patient charts. Cumulative cardiopulmonary bypass- (CPBT), circulatory arrest- (CAT) and cross-clamp times (CCT) were recorded. The patients' oxygen saturations and blood pressures at the time of CMR (prior to general anesthesia, if applied), serum biomarkers of hepatic function and hemodynamic data from cardiac catheterization as long as performed within 6 months of the CMR, were also retrieved.

Synthetic liver dysfunction was defined as an albumin concentration below the normal range in patients without protein losing enteropathy (PLE) and/or an elevation of the international normalized ratio (INR) above 1.2 in patients who were not receiving warfarin. Metabolic liver dysfunction was indicated by any of the following values outside of the normal range for age: unconjugated bilirubin, aspartate transaminase, alanine aminotransferase, alkaline phosphatase, gamma-glutamyl transferase, lactate dehydrogenase.

As a control group, patients examined between April 2014 and March 2017, with the same T1 relaxometry protocol for a family history of arrhythmogenic right ventricular cardiomyopathy or non-specific chest pain referred for anatomical coronary artery imaging were included, as long as their entire work-up was negative.

\section{Cardiovascular magnetic resonance imaging}

All patients and controls underwent CMR examinations on a $1.5 \mathrm{~T}$ system ('Magnetom Avanto', Siemens Healthineers, Erlangen, Germany). The detailed protocol has been published elsewhere [9]. In brief, a modified LookLocker inversion recovery (MOLLI) sequence at a single cardiac short axis slice was performed before and $15 \mathrm{~min}$ after injection of a gadolinium-based contrast agent, 0.2 $\mathrm{mmol} / \mathrm{kg}$ of either Gadobuturol ('Gadavist', Bayer Healthcare, Berlin, Germany) or gadobenate dimeglumine ('Multihance', Bracco S.p.A., Milan, Italy). A stack of balanced steady-state-free-precession short axis cine loops were obtained for volumetric measurements, in addition to through plane phase contrast cine acquisitions for blood flow measurements of the ascending and descending aorta and the pulmonary arteries and veins.

\section{Image post-processing}

All T1 mapping analyses were conducted by a single observer $(\mathrm{CdL})$. T1 relaxation times were extracted from the MOLLI acquisitions using commercially available software ('Qmap' in 'MedisSuite'version 2.1, MEDIS Medical Imaging Systems, Leiden, The Netherlands): Corresponding regions of interest (ROIs) were drawn on each of the eight motion corrected source images. T1 times were derived based on post-inversion recovery times and signal intensities, using a curve fitting algorithm.

Portions of the right and left lobes of the liver were visible on the mid-ventricular short axis slice. T1 relaxation times were measured by tracing three regions of interest (ROI): ROI 1, near the diaphragm, ROI 2, in the middle near the hepatic hilum, and ROI 3, in the caudal and peripheral part of the liver. All ROIs were traced to avoid major vessels, the gall bladder and hepatic surface (Fig. 1) [13, 14]. For blood pool T1 measurements, ROIs were placed in the cardiac lumen and in a hepatic vein as close as possible to the diaphragm, while avoiding myocardial trabeculations and hepatic tissue, respectively. All T1 measurements were corrected for incomplete inversion during the MOLLI acquisitions [15]. The ECV of all liver ROIs were calculated based on the preand post-contrast T1 values. For the blood pool T1 values, a $75 / 25 \%$ split between hepatic venous blood and cardiac lumen blood was used, given that $75 \%$ of hepatic blood flow is derived from the portal vein, i.e. is deoxygenated, similar to the hepatic venous blood. Hematocrit values were sampled within one week of the CMR examination (typically on the same day), and ECV was calculated according to the following formula :

$E C V=(1-$ haematocrit $) \frac{(1 / \text { post contrast } \mathrm{T} 1 \text { liver })-(1 / \text { native } \mathrm{T} 1 \text { liver })}{(1 / \text { post contrast } \mathrm{T} 1 \text { blood })-(1 / \text { native } \mathrm{T} 1 \text { blood })}$

A randomly selected sample of 20 patients was contoured by a second observer (MR) for observer variability. The same observer re-analyzed the same sample after an interval of at least 5 weeks blinded to the original results.

The same software platform ('MedisSuite,' 'Qmass' module) was used for the myocardial volumetric measurements: both ventricular chambers were contoured as one chamber as long as they were connected via a 


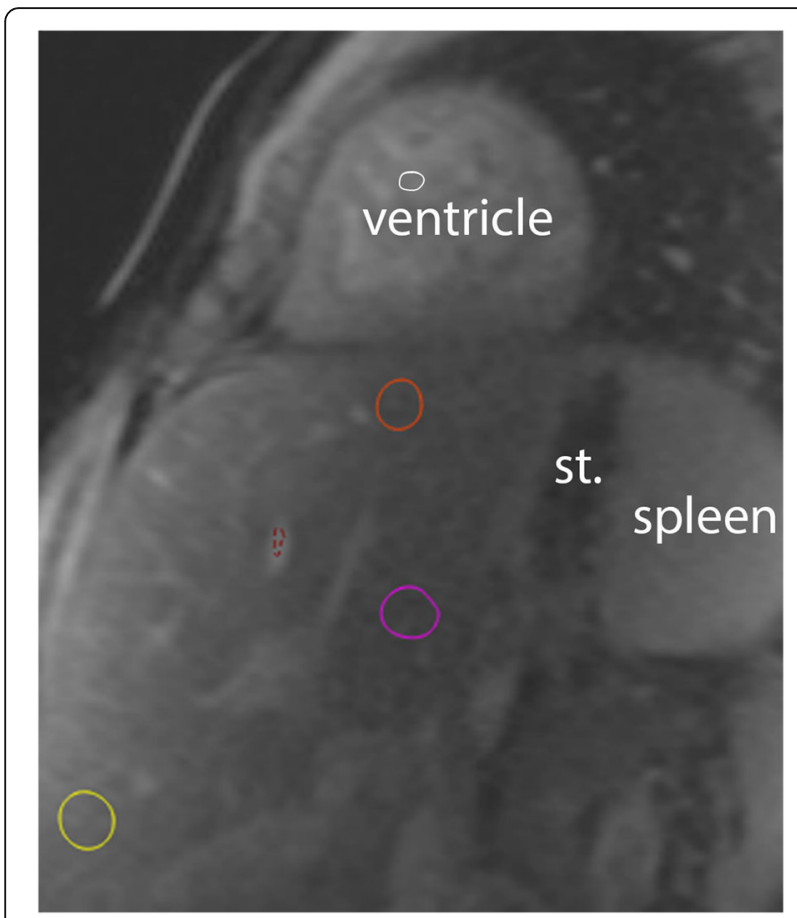

Fig. 1 Hepatic Native T1. Native T1 source image in short-axis orientation demonstrating the heart and upper part of the abdomen. Regions of interest (ROls) depict liver regions near diaphragm (\#1, orange), central (\#2, pink) and caudal (\#3, yellow). The red $\mathrm{ROI}$ samples signal in the blood pool of a liver vein and the white $\mathrm{ROI}(\# 5)$ in the ventricular cavity. Stomach (st)

ventricular septal defect (VSD) and / or both chambers ejected into the (neo-) aorta. Otherwise, only the dominant ventricle was contoured.

In addition, flow in the ascending and descending aorta, superior vena cava and the pulmonary arteries and veins were analyzed. Aorto-pulmonary collateral (APC) flow was calculated by subtracting total pulmonary arterial flow from the sum of the pulmonary venous flows.

\section{Statistical analysis}

Continuous variables are presented as means \pm standard deviation (SD) if normally distributed and otherwise as medians and ranges. Categorical variables are expressed as counts and percentages of the total. Student's t tests, Wilcoxon Rank-Sum tests or and analysis of variance (ANOVA) with post-hoc Tukey test for multiple comparisons, when appropriate, were used for comparisons between patient groups for continuous variables and Fisher's exact tests for categorical variables.

Correlations between continuous and categorical parameters were assessed using univariate regression analysis. A multivariate regression model was performed for variables with a significant number of patients and with $p$-values $<0.01$ on univariate testing. $P$-values $<0.05$ were considered statistically significant. Intra- and inter observer reproducibility's were assessed by BlandAltman analyses [16]. Statistical analyses were performed using SPSS version 25 (Statistical Package for the Social Sciences (SSPS) International Business Machines, Inc., Armonk, New York, USA).

\section{Results}

\section{Patient characteristics}

Patient characteristics including clinical parameters are presented in Table 1. Of 83 identified patients that met the inclusion criteria, 20 with BCPC $(2.8 \pm 0.9$ years, range $0.6-4.5)$ and 62 with Fontan $(11.4 \pm 4.4$ years, range $3.6-17.7)$ were included. One patient was excluded because of a non-diagnostic MOLLI sequence in whom only a small area of the liver was shown on the MOLLI images. In the Fontan group, there were 34 patients with a dominant single left ventricle (SLV) and 21 with a dominant single right ventricle (SRV). Seven patients had two good-sized ventricles and were excluded from analyses comparing SRV and SLV. In the BCPC group there were six patients with a SLV and ten with a SRV. Two patients had two good-sized ventricles and two had one single ventricle with unidentified morphological features. Forty-four individuals (age 13.9 \pm 2.7 years, range 9-18) constituted the control group.

Serum liver function testing was available in 31 Fontan patients and only two had all hepatic biomarkers within the normal range. None of the patients had severe metabolic or synthetic dysfunction. Twenty-three of 31 Fontan patients (74\%) had mildly abnormal parameters of metabolic hepatic function as evidenced by bilirubin, alkaline phosphatase, aspartate aminotransferase, lactate dehydrogenase and gamma-glutamyl transferase. Fourteen of 27 Fontan patients (52\%) had mildly deranged synthetic function with increase in INR (up to $1.8)$ and decrease in albumin (24-46 g/L, normal values $35-52 \mathrm{~g} / \mathrm{L})$.

\section{$\mathrm{T} 1$ and extracellular volume fraction}

Native T1 and ECV differed between the three ROIs (Additional file 1: Table S1). Given these differences, the average T1 and ECV from ROIs 1-3 were chosen as the most representative values for the liver and used for all subsequent analyses.

Blood pool native T1 values, were significantly higher in the ventricular blood pool than in hepatic venous blood, for controls $(1559 \pm 74$ and $1367 \pm 252 \mathrm{~ms}$ respectively, $p<0.001)$ and for patients $(1590 \pm 112$ versus $1427 \pm 207 \mathrm{~ms}$ respectively, $(p<0.001)$. This difference was attributed to the lower oxygen saturations in hepatic venous as compared to systemic ventricular blood.

Thirty-two out of 82 patients received gadobenate dimeglumine ('Multihance') and the remaining 50 patients (33 





Fontan and 17 BCPC patients) had gadobuturol ('Gadavist'). Of the 19 controls who received gadolinium, 15 had gadobenate dimeglumine and four gadobuturol. Liver ECV was markedly higher in subjects who had received gadobenate diglumine as compared to gadobuturol: $45.4 \pm 4.0 \%$ vs. $39.8 \pm 5.2 \%(p<0.001)$ for patients (BCPC and Fontan combined) and $58.1 \pm 12.6 \%$ and $32.1 \pm 4.8 \%$ $(p<0.001)$ for controls. For controls, the two groups did not differ significantly with regards to their gender, age composition or their hepatic native T1 times $(620 \pm 48$ and $610 \pm 71 \mathrm{~ms}$, respectively, $p=0.8$ ). We suspect that the ECV measurements in the gadobenate dimeglumine group were falsely elevated due to the partial biliary excretion (3-5\%) of the drug, and consequently, ECV was computed only for patients who had received gadobuturol.

Native T1 times in BCPC and Fontan patients were elevated as compared to controls, $(p<0.001$ for both groups) (Fig. 2 and Table 2). Native T1 and ECV were higher in Fontan than in BCPC patients ( $p=0.04$ for native $\mathrm{T} 1$ and ECV $p=0.02$, respectively). ECV was not significantly different for SLV and SRV $(p=0.1)$ (Table 2) . BCPC patients with SRVs had lower T1 values than SRV Fontan patients $(666 \pm 41$ versus $780 \pm 78 \mathrm{~ms}, p=<$ 0.001). Both T1 and ECV were lower in SLV BCPC patients than in SLV Fontan patients $(642 \pm 37$ versus $748 \pm 51 \mathrm{~ms}, p<0.001$ and $(36.1 \pm 4.8 \%$ versus $41.7 \pm$ $5.1 \%, p=0.001$, respectively).

The comparisons between Fontan patients with normal and abnormal hepatic (synthetic or metabolic) hepatic function regarding $\mathrm{T} 1$ and $\mathrm{ECV}$ were underpowered due to the low number of patients with available biochemistry data.

\section{Association between T1, ECV and clinical parameters}

In Fontan patients, $\mathrm{T} 1$ values correlated weakly with the sum of all previous CPBTs ( $\mathrm{R}=0.3 p=0.02$, CI (0.04-0, $40)$ ), with total CCT $(\mathrm{R}=0.3 p=0.03$, CI $(0.00-0.56))$ and with total CAT $(\mathrm{R}=0.3 \mathrm{p}=0.02$, CI $(-1.17-3.85))$.

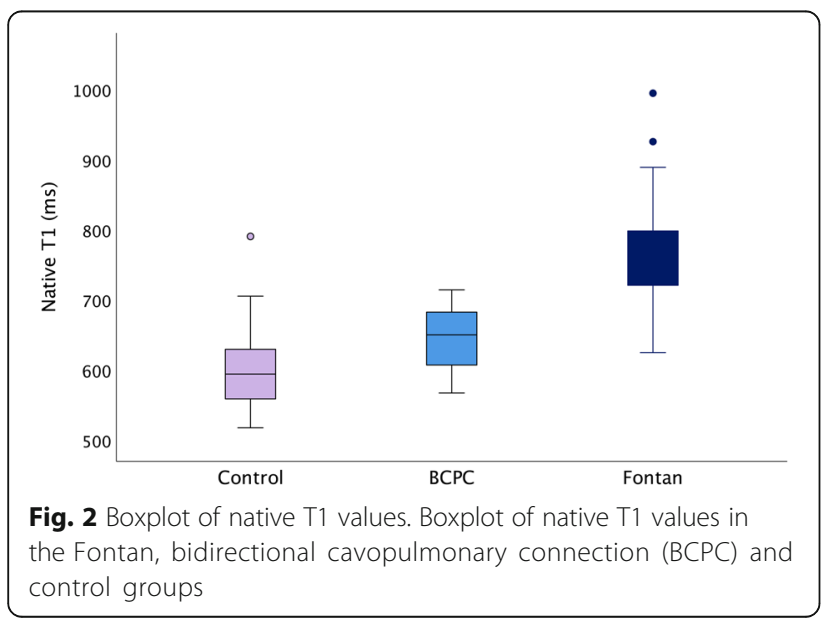

Native T1 correlated with end-systolic and end-diastolic volumes $(\mathrm{R}=0.3, p=0.04$ for both, $\mathrm{CI}(0.01-1.06)$ and (0.01-0.8) respectively) and with APC flow $(\mathrm{R}=0.3, p=$ 0.01 , CI (9.2-77.5)). Native T1 correlated inversely with $\mathrm{SaO}_{2}$ and body surface area $(\mathrm{R}=-0.3, \mathrm{p}=0.04$ for both, $\mathrm{CI}(-6.63--0.26)$ and $(-89.50--3.35)$ respectively).

There were no correlations of liver T1 or ECV with ventricular end-diastolic, and central venous pressures. However, the statistical power for these analyses was low due to the modest sample size. Patients with a patent Fontan fenestration $(n=44,70 \%)$ had similar $\mathrm{T} 1$ and $\mathrm{ECV}$ as the remainder of Fontan patients.

\section{Reproducibility}

Reproducibility was tested with Bland Altman plots for the T1 and ECV values of ROI 1-3. Native T1 and ECV demonstrated moderate intra- and inter-observer variation, with a relative bias of 0.1 and $0.2 \%$, respectively for $\mathrm{T} 1$ and $3.2 \%$ and $-2.3 \%$, respectively for ECV (Fig. 3). Coefficients of variation were 2.3 and 2.8 , respectively, for T1 and 10.7 and 10.9, respectively, for ECV. The reproducibility metrics of the individual ROIs are presented in Additional file 1: Table S1. In summary, the average of ROI 1-3 values which were considered the most representative for the displayed section of the liver tissue, revealed the best reproducibility.

\section{Discussion}

Liver fibrosis is an increasingly recognized complication in older Fontan patients but little is known about the severity of hepatic fibrotic remodeling in young individuals with single ventricle physiology. CMR T1 relaxometry presents an opportunity to measure the degree of extracellular volume expansion non-invasively. The current study helped us refine the technique of hepatic T1 relaxometry, shaping the methods in future studies. Our results offer the following novel insights into hepatic tissue changes:

1. CMR T1 mapping is feasible in the liver of young patients with congenital heart disease.

2. Imaging biomarkers of hepatic diffuse fibrosis and/ or congestion are elevated in young patients with single ventricle physiology, especially in those with a Fontan palliation.

3. Native $\mathrm{T} 1$ and ECV values are associated with exposure to cardiopulmonary bypass.

4. Technical advances from this study regarding hepatic ECV quantification are that a) gadolinium contrast agent with even partial biliary excretion must be avoided, b) hepatic venous and left ventricular blood pool T1values differ and c) averaging multiple ROIs is a suitable strategy to characterize liver ECV and T1 in light of heterogeneously distributed tissue changes in the Fontan population. 


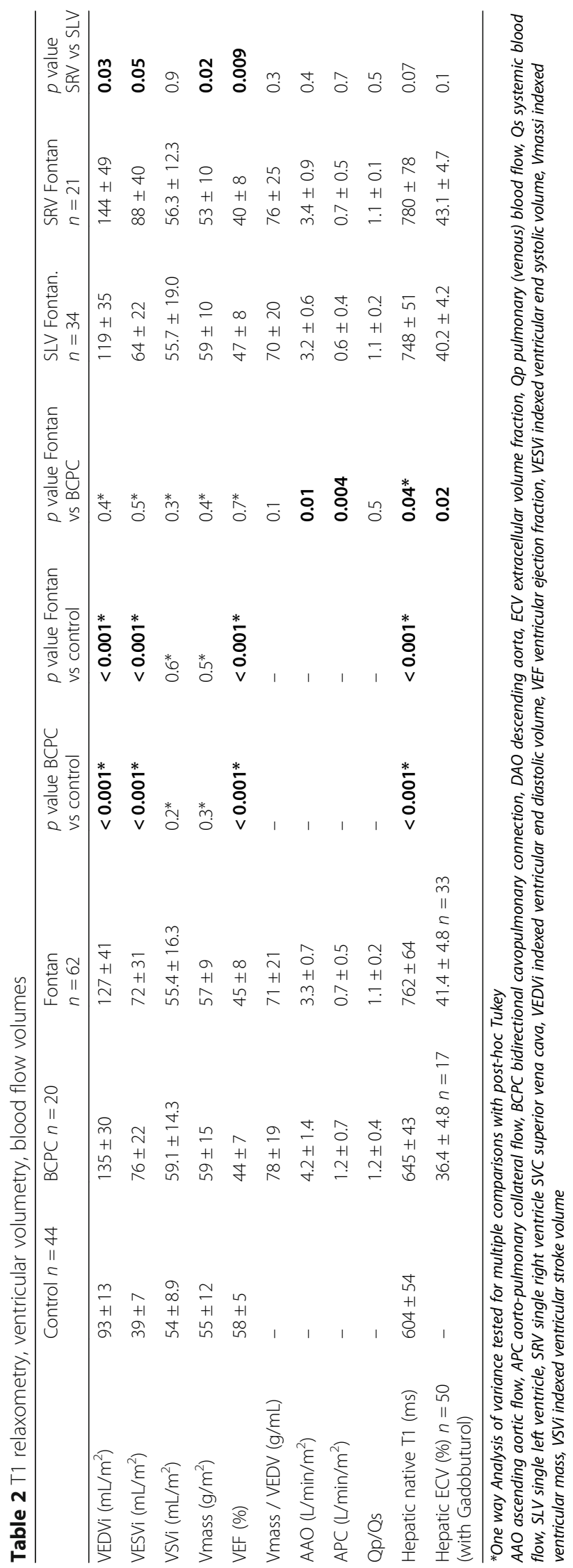



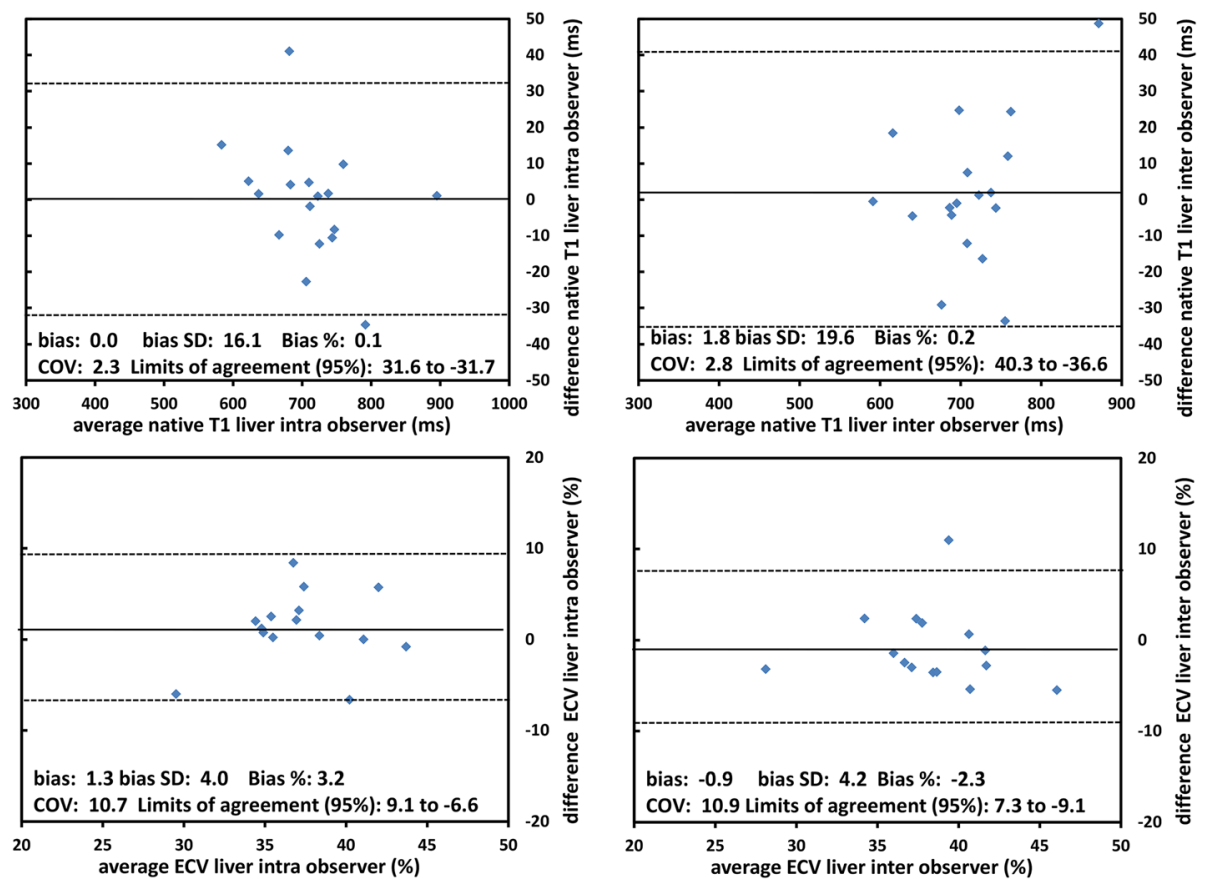

Fig. 3 Reproducibility of $\mathrm{T} 1$ and ECV. Bland Altman plots for intra and inter observer agreement for measurements of native $\mathrm{T} 1$ (upper row) and Extracellular volume fraction (ECV, lower row) for the average of ROls 1-3

\section{Fontan associated liver disease (FALD)}

FALD constitutes important morbidity in patients with functionally single ventricles. It affects candidacy for cardiac transplantation and other palliative operations. Furthermore, liver cirrhosis predisposes to malignant transformation of liver tissue to hepatocellular carcinoma [3, 17-21]. Early fibrotic remodeling, on the other hand, may be reversible and present a window for therapeutic intervention.

The challenge in monitoring hepatic fibrosis until recently lay within the lack of non-invasive techniques to detect and quantify the degree of remodeling. Liver biopsy is the diagnostic gold standard but invasive and not without risk. As such, it does not lend itself to serial examinations. Furthermore, due to the heterogeneous hepatic changes the sampled tissue may not be representative of the average degree of fibrosis $[22,23]$. More recently, 'stiffness' by elastography, based on either ultrasonography (USE) or magnetic resonance elastography (MRE), has assumed a role in the non-invasive evaluation of chronic liver disease, including in Fontan patients [21, 24-27]. However, the limitations of elastography are that it a) is an indirect measure of tissue behavior rather than a direct marker of extracellular volume expansion, b) does not distinguish between fibrosis and congestion, c) has not been demonstrated to predict outcome, and d) requires additional hardware not widely available.

\section{$\mathrm{T} 1$ and ECV are elevated in BCPC and Fontan patients}

CMR T1 relaxometry overcomes some of these limitations by providing non-invasive, quantitative tissue characterization with a spatial resolution in the millimeter range. In non-congestive adult chronic liver disease, native $\mathrm{T} 1$ times can discriminate between histologic fibrotic grades $[10,28,29]$ and may predict outcomes [11,30].

Only pilot data in 16 adult Fontan patients yielded CMR T1 and ECV levels comparable to our cohort [14]. In our study, Fontan patients demonstrated elevated native T1 times, in line with results from USE, computed tomography and postmortem studies which have indicated increased stiffness and evidence of fibrosis [3134]. Fontan patients had higher T1 and ECV than BCPC patients, suggesting an increase in fibrosis/ congestion as patients transition to the Fontan circulation. Whether this is true in individual patients remains to be confirmed with serial CMRs. A recent USE study revealed a transient increase in hepatic stiffness soon after the Fon$\tan$ operation, leading the authors to hypothesize that in this scenario stiffness represents congestion, rather than fibrosis which would take longer to develop [26]. Our results did not reveal an association of either T1 or ECV with central venous pressure (CVP). However, these analyses were poorly powered due to the modest sample size. Like elastography, T1 relaxometry does not distinguish unequivocally between fibrosis, congestion or 
inflammation, as all elevate both $\mathrm{T} 1$ and $\mathrm{ECV}$; it seems plausible that all three mechanisms contribute to the abnormal $\mathrm{T} 1$ and ECV values. In the future, adding T2 mapping and $\mathrm{T} 2$ weighted imaging may prove helpful in distinguishing between fibrosis and edema. Further, T1rho relaxation time may become useful in monitoring liver fibrosis and in distinguishing it from fat, edema and/or inflammation [22, 35, 36]. Despite an uncertainty about the exact histological correlate, monitoring markers of extracellular matrix expansion may prove beneficial, given that congestion is regarded as a precursor of FALD [37-39]. Nonetheless, the prognostic significance of liver $\mathrm{T} 1$ relaxometry remains to be determined.

Our results suggest that the etiology of hepatic changes is multifactorial: For example, exposure to cardiopulmonary bypass is associated not only with longterm myocardial, but also hepatic remodeling [9, 40]. Another candidate risk factor for hepatic remodeling are decreased oxygen delivery as a result of hypoxemia and low cardiac output in this population. An association between CVP and liver stiffness was mostly described in older Fontan patient populations. In our young cohort, the other potential factors may be relatively more important than CVP. In this context, it is interesting to note that T1 and ECV did not increase with age, also pointing to factors other than chronic venous congestion as the principal pathomechanism [34].

Fontan patients with an SRV revealed a trend towards higher liver T1 values than those with a SLV. Whether this difference is related to longer bypass and crossclamp times, the worse ventricular performance or a less obvious risk factor in the SRV group remains to be determined.

\section{$\mathrm{T} 1, \mathrm{ECV}$ and liver function}

In keeping with other pediatric reports, markers of hepatic function revealed only mild perturbations of synthetic and metabolic performance in 52 and $74 \%$, respectively, of the Fontan patients who underwent biochemistry testing. No reliable analysis of an association of serum markers with ECV or T1 could be performed due to small sample size. Previous histological studies highlight the poor reflection of early liver injury in serum markers, due to the liver's remarkable ability to compensate for these tissue changes until their late stages [19, 33].

\section{Liver T1 Relaxometry - technical aspects}

The current study yields several important technical insights into liver T1 mapping and ECV analysis. Most importantly, gadolinium agents with even a small percentage of biliary secretion, such as gadobenate dimeglumine, are not suitable for the quantification of ECV because their concentration in the bile system falsely decreases post-contrast T1, leading to erroneously high ECV results.

In contrast to previously published reports [14] our data suggest that the blood pool $\mathrm{T} 1$ producing the most representative hepatic ECV measurements is not the ventricular cavity which is used for myocardial analyses. We found systematically found lower precontrast $\mathrm{T} 1$ times in the liver vein as compared to the heart. The principal cause of this discrepancy is likely the lower oxygen saturations in hepatic venous as compared to ventricular blood, as $\mathrm{T} 1$ is lower in less oxygenated blood [41]. In the liver, blood flow is mainly provided by the portal vein in about $75 \%$ while $25 \%$ is provided by the hepatic artery [39]. Therefore, performing the ECV calculations using a $75 / 25 \%$ split between hepatic venous blood and arterial cardiac lumen blood can be expected to produce a more 'true' hepatic ECV value.

Liver fibrosis is known to be a geographically heterogeneous process. As a result, we found systematic regional differences in the sampled T1. Previous studies implied that portal venous hypertension progresses centripetally from the liver periphery $[38,39]$. As such, one would expect lower fibrosis/congestion markers near the center (ROI1) which was true in our cohort. Given the observed regional variation of tissue composition along with the superior reproducibility of averaging multiple ROIs, sampling $\mathrm{T} 1$ in multiple regions is the preferred approach in the Fontan population [13, 14, 29]. Sampling a large ROI encompassing the entire liver in the field of view is problematic as it is challenging to avoid medium sized hepatic vessels.

The technical challenges with hepatic MR relaxometry pertain mostly to ECV and its calculation. In the present study, ECV did not appear to add insights beyond what was apparent from native T1 analysis. Relying solely on T1 and foregoing ECV is attractive as it avoids the need for intravenous gadolinium as well as abbreviate scan and postprocessing times. On the other hand, ECV has been demonstrated to be more comparable across scanner manufacturers, field strengths, and patient populations [37, 42]. It is too early to conclude whether liver MR relaxometry should be performed using native T1, ECV or both.

\section{Study limitations}

Several limitations of this study warrant discussion: 1.) Since, most control subjects received gadobenate dimeglumine which is biliarily excreted, there was no control group for hepatic ECV. 2.) The pediatric control group was older than the patient group, limiting the comparability between the groups. Furthermore, the $\mathrm{BCPC}$ group was more homogeneous in age than 
the Fontan group which may have accounted for broader range of $\mathrm{T} 1$ and ECV values among Fontan patients. This relatively wide spectrum may have obscured some of the differences between Fontan patients and controls. 3.) It is possible that postprandial conditions, not controlled for during this study, influences native T1 and ECV owing to differences in portal venous flow [43]. 4.) Given the heterogeneity of hepatic remodeling the section of the liver that was included in the short axis MOLLI slice may not be representative of the entire liver parenchyma. For prospective studies, dedicated $\mathrm{T} 1$ acquisitions of the liver are advised. While it would be preferable to obtain the $\mathrm{T} 1$ value for the entire portion of the liver displayed in the image this was not feasible due to the signal from blood vessels in the image which would have contaminated the T1 values and could not be excluded by thresholding due to imperfect coregistration of the source images despite motion correction. 5.) It is unclear whether axial liver T1 mapping acquisitions would have yielded different results from the short axis sequences that were available for review. 6.) Even though we used a blood pool signal with an oxygen saturation that approximated that within the liver parenchyma for $\mathrm{T} 1$, the resultant ECV may differ from the true liver values. 7.) Low albumin, which was used as a marker of hepatic synthetic insufficiency, also occurs in PLE. While this parameter was not evaluated in patients with overt PLE, an intestinal protein leak can be subclinical in Fontan patients. 8.) The retrospective study design may have introduced a selection bias and caused gaps in some of the results, most notably in the liver serum biomarkers, which were available only in a subgroup of patients. 9.) Finally, while the crosssectional information presented here suggests that fibrosis / congestion markers increase with the transition from the BCPC to the Fontan circulation only longitudinal data can elucidate the development of these changes in individual patients over time.

\section{Conclusion}

In this study of a large cohort of pediatric patients with single ventricle circulations, imaging biomarkers of fibrosis and/or congestion are elevated and particularly after the Fontan operation. These liver tissue changes are not clearly associated with Fontan pressure or ventricular systolic performance. Longitudinal studies are required to test the prognostic significance of these derangements. Pitfalls of ECV quantification are related to the use of gadolinium contrast with biliary excretion, blood pool T1 sampling regions, and the heterogeneity of liver remodeling.

\section{Additional file}

Additional file 1: Table S1. Inter region T1, ECV differences. Native T1 times and extracellular volume fraction (ECV) for the different ROls 1-3 and the average of ROls 1-3 with observer variation. (DOC $41 \mathrm{~kb}$ )

\section{Abbreviations \\ ANOVA: Analysis of variance; APC: Aortopulmonary collateral; BCPC: Bidirectional cavopulmonary connection; CAT: Circulatory arrest time; CCT: Cross-clamp time; CMR: Cardiovascular magnetic resonance; CPBT: Cardiopulmonary bypass time; CVP: Central venous pressure; ECV: Extracellular volume fraction; EF: Ejection fraction; FALD: Fontan associated liver disease; INR: International normalized ratio; LGE: Late gadolinium enhancement; MOLLI: Modified Look-Locker inversion recovery; MRE: Magnetic resonance elastography; PLE: Protein losing enteropathy; ROI: Region of interest; SD: Standard deviation; SLV: Single left ventricle; SRV: Single right ventricle; SV: Single ventricle; USE: Ultrasonography elastography; VEDP: Ventricular end diastolic pressure; VEDV: Ventricular end diastolic volume; VESV: Ventricular end systolic volume; VSD: Ventricular septal defect}

\section{Acknowledgements}

We thank Chun-Po Steve Fan, PhD, for his contributions to the statistical analysis.

\section{Authors' contributions}

$\mathrm{CDL}$ analyzed and collected data, assisted with study design and implementation, and drafted the manuscript. MR analyzed and collected data, and assisted with critical revision of the manuscript. $\mathrm{CL}, \mathrm{JP}$ collected data, and assisted with critical revision of the manuscript. SJY MS and CB assisted with interpretation of data, and critical revision of the manuscript for important intellectual content. LGW conceived the study, assisted in study design, in data collection, and assisted with drafting and critical revision of the manuscript. All authors read and approved the final manuscript.

\section{Funding}

None

\section{Availability of data and materials}

The datasets used and/or analyzed during the current study are available from the corresponding author on reasonable request.

\section{Ethics approval and consent to participate}

This retrospective study was approved by the institutional research ethics board which waived the requirement for written informed consent.

\section{Consent for publication}

Not applicable.

\section{Competing interests}

The authors declare that they have no competing interests.

\section{Author details}

${ }^{1}$ Department of Pediatrics, Division of Cardiology, The Hospital for Sick Children, University of Toronto, Ontario, Canada. ${ }^{2}$ Division of Radiology and Nuclear medicine, Pediatric section, Rikshospitalet, Oslo University Hospital, Oslo, Norway. ${ }^{3}$ Department of Pediatrics, Division of Cardiology, Stollery Children's Hospital, University of Alberta, Edmonton, Alberta, Canada. ${ }^{4}$ Department of Diagnostic Imaging, The Hospital for Sick Children, University of Toronto, Toronto, Ontario, Canada. ${ }^{5}$ Knight Cardiovascular Institute, Division of Cardiovascular Medicine, Oregon Health and Science University, Portland, Oregon, USA.

Received: 23 October 2018 Accepted: 28 May 2019 Published online: 15 July 2019

\section{References}

1. Khairy P, Poirier N, Mercier LA. Univentricular heart. Circulation. 2007;115: 800-12. 
2. Fontan F, Baudet E. Surgical repair of tricuspid atresia. Thorax. 1971;26:240-8.

3. Mori M, Aguirre AJ, Elder RW, Kashkouli A, Farris AB, Ford RM, et al. Beyond a broken heart: circulatory dysfunction in the failing Fontan. Pediatr Cardiol. 2014;35:569-79

4. Rychik J. Forty years of the Fontan operation: a failed strategy. Semin Thorac Cardiovasc Surg Pediatr Card Surg Annu. 2010;13:96-100.

5. Broberg CS, Chugh SS, Conklin C, Sahn DJ, Jerosch-Herold M. Quantification of diffuse myocardial fibrosis and its association with myocardial dysfunction in congenital heart disease. Circ Cardiovasc Imaging. 2010;3:727-34.

6. Babu-Narayan SV, Kilner PJ, Li W, Moon JC, Goktekin O, Davlouros PA, et al. Ventricular fibrosis suggested by cardiovascular magnetic resonance in adults with repaired tetralogy of fallot and its relationship to adverse markers of clinical outcome. Circulation. 2006;113:405-13.

7. Riesenkampff E, Messroghli DR, Redington AN, Grosse-Wortmann L. Myocardial T1 mapping in pediatric and congenital heart disease. Circ Cardiovasc Imaging. 2015;8(2):e002504. https://doi.org/10.1161/ CIRCIMAGING.114.002504.

8. Dusenbery SM, Jerosch-Herold M, Rickers C, Colan SD, Geva T, Newburger JW, et al. Myocardial extracellular remodeling is associated with ventricular diastolic dysfunction in children and young adults with congenital aortic stenosis. J Am Coll Cardiol. 2014;63:1778-85.

9. Kato A, Riesenkampff E, Yim D, Yoo SJ, Seed M, Grosse-Wortmann L. Pediatric Fontan patients are at risk for myocardial fibrotic remodeling and dysfunction. Int J Cardiol. 2017;240:172-7.

10. Cassinotto C, Feldis M, Vergniol J, Mouries A, Cochet H, Lapuyade B, et al. MR relaxometry in chronic liver diseases: comparison of T1 mapping, T2 mapping, and diffusion-weighted imaging for assessing cirrhosis diagnosis and severity. Eur J Radiol. 2015;84:1459-65.

11. Banerjee R, Pavlides M, Tunnicliffe EM, Piechnik SK, Sarania N, Philips R, et al. Multiparametric magnetic resonance for the non-invasive diagnosis of liver disease. J Hepatol. 2014;60:69-77.

12. Yoon JH, Lee JM, Paek M, Han JK, Choi Bl. Quantitative assessment of hepatic function: modified look-locker inversion recovery (MOLLI) sequence for T1 mapping on Gd-EOB-DTPA-enhanced liver MR imaging. Eur Radiol. 2016;26:1775-82.

13. Haimerl M, Utpatel K, Verloh N, Zeman F, Fellner C, Nickel D, et al. Gd-EOBDTPA-enhanced MR relaxometry for the detection and staging of liver fibrosis. Sci Rep. 2017;7:41429

14. Kazour I, Serai SD, Xanthakos SA, Fleck RJ. Using T1 mapping in cardiovascular magnetic resonance to assess congestive hepatopathy. Abdom Radiol (NY). 2018;43:2679-85.

15. Kellman P, Herzka DA, Hansen MS. Adiabatic inversion pulses for myocardial T1 mapping. Magn Reson Med. 2014;71:1428-34.

16. Bland JM, Altman DG. Statistical methods for assessing agreement between two methods of clinical measurement. Lancet. 1986;1:307-10.

17. Greenway SC, Crossland DS, Hudson M, Martin SR, Myers RP, Prieur T, et al. Fontan-associated liver disease: implications for heart transplantation. J Heart Lung Transplant. 2016;35:26-33.

18. Rychik J. The relentless effects of the Fontan paradox. Semin Thorac Cardiovasc Surg Pediatr Card Surg Annu. 2016;19:37-43.

19. Daniels CJ, Bradley EA, Landzberg MJ, Aboulhosn J, Beekman RH 3rd, Book $W$, et al. Fontan-associated liver disease: proceedings from the American College of Cardiology Stakeholders Meeting, October 1 to 2, 2015, Washington DC. J Am Coll Cardiol. 2017;70:3173-94.

20. Nandwana SB, Olaiya B, Cox K, Sahu A, Mittal P. Abdominal imaging surveillance in adult patients after Fontan procedure: risk of chronic liver disease and hepatocellular carcinoma. Curr Probl Diagn Radiol. 2018;47:19-22.

21. Poterucha JT, Johnson JN, Qureshi MY, O'Leary PW, Kamath PS, Lennon RJ, et al. Magnetic resonance Elastography: a novel technique for the detection of hepatic fibrosis and hepatocellular carcinoma after the Fontan operation. Mayo Clin Proc. 2015;90:882-94.

22. Unal E, Idilman IS, Karcaaltincaba M. Multiparametric or practical quantitative liver MRl: towards millisecond, fat fraction, kilopascal and function era. Expert Rev Gastroenterol Hepatol. 2017;11:167-82.

23. Poynard T, Lenaour G, Vaillant JC, Capron F, Munteanu M, Eyraud D, et al. Liver biopsy analysis has a low level of performance for diagnosis of intermediate stages of fibrosis. Clin Gastroenterol Hepatol. 2012;10:657-63.

24. Serai SD, Wallihan DB, Venkatesh SK, Ehman RL, Campbell KM, Sticka J, et al. Magnetic resonance elastography of the liver in patients status-post fontan procedure: feasibility and preliminary results. Congen Heart Dis. 2014;9:7-14.
25. Melero-Ferrer JL, Osa-Saez A, Buendia-Fuentes F, Ballesta-Cunat A, Flors L, Rodriquez-Serrano $\mathrm{M}$, et al. Fontan circulation in adult patients: acoustic radiation force impulse Elastography as a useful tool for liver assessment. World J Pediatr Congenit Heart Surg. 2014;5:365-71.

26. DiPaola FW, Schumacher KR, Goldberg CS, Friedland-Little J, Parameswaran A, Dillman JR. Effect of Fontan operation on liver stiffness in children with single ventricle physiology. Eur Radiol. 2017;27:2434-42.

27. Trout AT, Sheridan RM, Serai SD, Xanthakos SA, Su W, Zhang B, et al. Diagnostic performance of MR Elastography for liver fibrosis in children and Young adults with a Spectrum of liver diseases. Radiology. 2018;287:824-32.

28. Heye T, Yang SR, Bock M, Brost S, Weigand K, Longerich T, et al. MR relaxometry of the liver: significant elevation of $\mathrm{T} 1$ relaxation time in patients with liver cirrhosis. Eur Radiol. 2012;22:1224-32.

29. Kim KA, Park MS, Kim IS, Kiefer B, Chung WS, Kim MJ, et al. Quantitative evaluation of liver cirrhosis using T1 relaxation time with 3 tesla MRI before and after oxygen inhalation. J Magn Reson Imaging. 2012;36:405-10.

30. Pavlides M, Banerjee R, Sellwood J, Kelly CJ, Robson MD, Booth JC, et al. Multiparametric magnetic resonance imaging predicts clinical outcomes in patients with chronic liver disease. J Hepatol. 2016;64:308-15.

31. Baek JS, Bae EJ, Ko JS, Kim GB, Kwon BS, Lee SY, et al. Late hepatic complications after Fontan operation; non-invasive markers of hepatic fibrosis and risk factors. Heart. 2010;96:1750-5.

32. Friedrich-Rust M, Koch C, Rentzsch A, Sarrazin C, Schwarz P, Herrmann E, et al. Noninvasive assessment of liver fibrosis in patients with Fontan circulation using transient elastography and biochemical fibrosis markers. J Thorac Cardiovasc Surg. 2008;135:560-7.

33. Hilscher MB, Johnson JN, Cetta F, Driscoll DJ, Poterucha JJ, Sanchez W, et al. Surveillance for liver complications after the Fontan procedure. Congenit Heart Dis. 2017;12:124-32.

34. Schwartz MC, Sullivan L, Cohen MS, Russo P, John AS, Guo R, et al. Hepatic pathology may develop before the Fontan operation in children with functional single ventricle: an autopsy study. J Thorac Cardiovasc Surg. 2012; 143:904-9.

35. Zhang H, Yang Q, Yu T, Chen X, Huang J, Tan C, et al. Comparison of T2, T1rho, and diffusion metrics in assessment of liver fibrosis in rats. J Magn Reson Imaging. 2017:45:741-50.

36. Xie S, Li Q, Cheng Y, Zhang Y, Zhuo Z, Zhao G, et al. Impact of liver fibrosis and fatty liver on T1 rho measurements: a prospective study. Korean $J$ Radiol. 2017;18:898-905.

37. Schelbert EB, Messroghli DR. State of the art: clinical applications of cardiac T1 mapping. Radiology. 2016;278:658-76.

38. Wells ML, Fenstad ER, Poterucha JT, Hough DM, Young PM, Araoz PA, et al. Imaging findings of congestive Hepatopathy. Radiographics. 2016;36:1024-37.

39. Yoo SJ, Prsa M, Schantz D, Grosse-Wortmann L, Seed M, Kim TK, et al. MR assessment of abdominal circulation in Fontan physiology. Int J Cardiovasc Imaging. 2014;30:1065-72.

40. Yim D, Riesenkampff E, Caro-Dominguez P, Yoo SJ, Seed M, Grosse-Wortmann L. Assessment of Diffuse Ventricular Myocardial Fibrosis Using Native T1 in Children With Repaired Tetralogy of Fallot. Circ Cardiovasc Imaging 2017;10 pii: e005695. https://doi.org/10.1161/CIRCIMAGING.116.005695.

41. Portnoy S, Osmond M, Zhu MY, Seed M, Sled JG, Macgowan CK. Relaxation properties of human umbilical cord blood at 1.5 tesla. Magn Reson Med. 2017;77:1678-90.

42. Messroghli DR, Moon JC, Ferreira VM, Grosse-Wortmann L, He T, Kellman P, et al. Clinical recommendations for cardiovascular magnetic resonance mapping of $T 1, T 2, T 2^{*}$ and extracellular volume: a consensus statement by the Society for Cardiovascular Magnetic Resonance (SCMR) endorsed by the European Association for Cardiovascular Imaging (EACVI). J Cardiovasc Magn Reson. 2017;19:75. https://doi.org/10.1186/s12968-017-0389-8.

43. Palaniyappan N, Cox E, Bradley C, Scott R, Austin A, O'Neill R, et al. Noninvasive assessment of portal hypertension using quantitative magnetic resonance imaging. J Hepatol. 2016;65:1131-9.

\section{Publisher's Note}

Springer Nature remains neutral with regard to jurisdictional claims in published maps and institutional affiliations. 